\title{
A influência da amamentação no desenvolvimento emocional infantil: percepções maternas
}

\author{
The influence of breastfeeding in child emotional development: maternal perceptions \\ La Influencia de la lactancia en el desarrollo emocional infantil: percepciones de las madres \\ Carina Camilo Lima Pedro ${ }^{1}$, Aurea Tamami Minagawa Toriyama ${ }^{1}$
}

\begin{abstract}
Resumo
Objetivo: Descrever o conhecimento produzido a respeito das percepções maternas sobre a influência da amamentação no desenvolvimento emocional infantil.

Métodos: Revisão integrativa em bases de dados notórias. Realizou-se análise de conteúdo dos resultados e definição de categorias temáticas.

Resultados: Foram analisados 13 artigos que evidenciaram as percepções maternas sobre amamentação, fatores de desmame, fatores que influenciam a manutenção da prática, sentimentos decorrentes, orientações recebidas e influência no desenvolvimento infantil. Há uma única publicação que trata sobre as percepções maternas sobre a influência da amamentação no desenvolvimento emocional infantil.

Conclusão: A abordagem das percepções maternas sobre a influência da amamentação no desenvolvimento emocional das crianças é escassa na literatura científica.
\end{abstract}

\section{Abstract}

Objective: To describe knowledge produced about maternal perceptions about the influence of breastfeeding on child emotional development.

Methods: Integrative review in recognized databases. Realized Content Analysis of the Results and defined thematic categories.

Results: 13 articles have evidenced the maternal perceptions about breastfeeding, weaning factors, factors that influence the maintenance of the practice, feelings, guidelines received and influence of breastfeeding on child development. There is a single publication dealing with maternal perceptions about the influence of breastfeeding on infant emotional development.

Conclusion: The approach of maternal perception about the influence of breastfeeding on child emotional development is scarce in literature.

\section{Resumen}

Objetivo: Describir el conocimiento producido sobre las percepciones maternas sobre la influencia de la lactancia materna en el desarrollo emocional infantil.

Métodos: Revisión integrativa realizada en bases de datos notorias. Se realizó análisis de contenido de los resultados y definición de categorías temáticas.

Resultados: 13 artículos evidenciaron las percepciones maternas sobre lactancia, factores de destete, factores que influencian el mantenimiento de la práctica, sentimientos resultantes, orientaciones recibidas e influencia en el desarrollo infantil. Hay una única publicación que trata sobre las percepciones maternas sobre la influencia de la lactancia en el desarrollo emocional infantil.

Conclusión: El enfoque de las percepciones maternas en la influencia de la lactancia en el desarrollo emocional de los niños es escaso en la literatura científica.

\section{Descritores}

Amamentação; Desenvolvimento infantil; Percepções maternas

\section{Keywords}

Breastfeeding; Child development;

Maternal perceptions

\section{Descriptres}

Lactancia; Desarrollo infantil;

Percepciones de las madres

\section{Como citar:}

Pedro CC, Toriyama AT. [The influence of breastfeeding in child emotional development: maternal perceptions]. Rev Soc Bras Enferm Ped. 2018;18(2):103-8. Portuguese

${ }^{1}$ Escola de Enfermagem, Universidade de São Paulo, São Paulo, SP, Brasil.

Conflitos de interesse: nada a declarar.

Submissão: 2 de Agosto de 2018| Aceite: 27 de Junho de 2019

Autor correspondente: Carina Camilo Lima Pedro | Av. Dr. Enéas de Carvalho Aguiar, 419, 05403-000, São Paulo, SP, Brasil. carina.lima.c@gmail.com

https://orcid.org/0000-0001-8979-6970

DOI: http://dx.doi.org/10.31508/1676-3793201800016 


\section{Introdução}

A amamentação promove a saúde da criança e diminui as taxas de morbimortalidade infantil, além de promover melhor rendimento escolar e maiores salários na idade adulta. ${ }^{(1-3)}$

A amamentação tem grande influência no desenvolvimento infantil, em suas três esferas: física (ou biológica), cognitiva e emocional (ou psicossocial). ${ }^{(4)} \mathrm{O}$ desenvolvimento físico é inerente à capacidade maturacional da criança crescer em tamanho e proporção. ${ }^{(5)}$ Já o desenvolvimento cognitivo é a capacidade intelectual da criança em atingir alguns marcos pertinentes a cada faixa etária, como aquisição de vocabulário, memória e linguagem. ${ }^{(6)}$

O desenvolvimento emocional infantil se reflete na capacidade em diferenciar emoções cada vez mais complexas e autorregulá-las. Ele pode ser percebido quando a criança, por exemplo, com quatro meses sorri para as expressões de um rosto, ou mexe as pernas, braços e barriga quando é tocado. Com oito meses estende os braços quando quer ser abraçado. Aos 12 meses, diante de uma dificuldade, fica bem (recupera-se de um fator estressante) após 10 ou 15 minutos. Com 18 meses, ou um ano e meio: pede ajuda aos pais para brincar ou comer; vai até a geladeira para mostrar a comida que quer. De um a dois anos, usa frases como "me abraça" e "eu quero", e demonstra raiva ou "birra", mas fica bem após 10 minutos. ${ }^{(7)}$

A amamentação pode influenciar no desenvolvimento emocional infantil, pois promove o vínculo entre a mãe e a criança. Assim, a mãe atua como agente primário de satisfações fisiológicas do filho, ${ }^{(8)}$ além de suprir a necessidade essencial da criança de manter um relacionamento sustentador contínuo e seguro. ${ }^{(9)}$

Estudo de coorte verificou que as mães que amamentaram de forma exclusiva até os seis meses tiveram maior sensibilidade ao filho quando comparado às mães que não amamentaram. Aquelas conseguiam realizar uma "leitura" das necessidades emocionais de seus filhos. ${ }^{(10)}$

Segundo estudo da Universidade de Yale, regiões cerebrais responsáveis pelas emoções de mães que amamentavam eram mais ativadas quando ouviam os choros de seus filhos em comparação com mães que alimentavam seus filhos por mamadeira. ${ }^{(11)}$ Essa interação frequente entre mãe e criança pode atuar como um influenciador do desenvolvimento emocional infantil até mesmo a longo prazo: estudo longitudinal realizado nos EUA verificou que crianças que haviam sido amamentadas nos primeiros seis meses tinham menos problemas de conduta e dificuldades gerais aos seis anos quando comparadas às crianças que haviam sido alimentadas por fórmula. ${ }^{(12)}$

Mesmo assim, a amamentação no Brasil ainda demanda investimentos diversos: segundo pesquisa nacional realizada em 2008, a mediana da duração da amamentação exclusiva é de apenas 1,8 meses e de aleitamento materno de 11,4 meses. ${ }^{(13)}$

Os determinantes do aleitamento materno são diversos, incluindo os psicossociais. Sabe-se que a atitude positiva e a intenção prévia de amamentar podem ser fatores correlacionados a um maior tempo de amamentação. A mãe que acredita que a amamentação é eficaz no desenvolvimento de seu filho parece conseguir manter a prática por mais tempo. Da mesma forma, uma mãe que tem o conhecimento de que o seu filho pode chorar não apenas por fome, mas também por necessidade de afeto, pode ter menor intenção de desmamar ao se deparar com o choro de sua criança. ${ }^{(14)}$

Assim, conhecer as percepções maternas a respeito da influência da amamentação no desenvolvimento emocional de seus filhos pode direcionar as intervenções dos profissionais de saúde. Buscou-se responder à seguinte pergunta: "Quais as percepções das mães a respeito da influência da amamentação no desenvolvimento emocional de seus filhos?". Dessa forma, verificar as lacunas existentes nesse âmbito e as evidências da influência da amamentação no cuidado em saúde poderá trazer sustentação à prática cotidiana dos profissionais.

\section{Métodos}

Revisão integrativa com as seguintes etapas: 1) Definição da questão norteadora; 2) Seleção da amostra; 3) Leitura integral dos estudos; 4) Avaliação dos estudos; 5)Categorização dos estudos; 6) Análise e Apresentação dos resultados; 7) Discussão dos resultados; 8) Síntese do conhecimento.

A pergunta para revisão integrativa foi: "Quais as percepções das mães a respeito da amamentação e do desenvolvimento emocional de seus filhos?". 
Não houve período delimitado para que a busca literária fosse a mais abrangente possível e os resultados aparecessem de forma livre. A pesquisa foi realizada nos seguintes bancos e bases de dados informatizados: Todas as bases de Dados do Portal Biblioteca Virtual em Saúde (BVS), Cumulative Index of Nursing and Allied Health Literature (CINAHL), SCOPUS, PsycINFO, Embase e portal PUBMED.

Foram incluídos na busca artigos de acesso on line relacionados ao tema de amamentação, desenvolvimento emocional infantil e percepções maternas, nos idiomas português, inglês e espanhol. As revisões da literatura, teses, monografias e dissertações foram excluídas. A busca foi realizada em quatro momentos diferentes e os termos utilizados em português foram: amamentação, aleitamento materno, vínculo, percepção materna, percepções maternas, opinião materna, opiniões maternas, desenvolvimento infantil, desenvolvimento emocional, desenvolvimento da personalidade. Em inglês foram: breast feeding, breastfeeding, breast-feeding, emotional development in early childhood, infant behavior, childhood behavior, emotional development, child emotional development, early childhood development, psychological development, mother's experience, mother, perceptions, mother child relations, motherchild relations, mother's experiences, mother-infant relations, maternal experiences, maternal perception, maternal perceptions, breast feeding experiences, breastfeeding experiences.

Foram lidos na íntegra os artigos condizentes pelos resumos e descartados os que não se relacionavam à temática. A seguir, foi realizada análise temática de conteúdo dos estudos com posterior formação de categorias.

\section{Resultados e Discussão}

A amostra final foi composta por 13 artigos. ${ }^{(15-27)}$ Em relação ao idioma, apenas cinco deles foram escritos em português, ${ }^{(15-17,26,27)}$ sendo os demais apresentados em língua inglesa. ${ }^{(18-25)}$ Em relação à nacionalidade, quatro artigos são brasileiros, ${ }^{(15,16,26,27)}$ um português, ${ }^{(17)}$ dois dos EUA, ${ }^{(18,19)}$ um inglês, ${ }^{(20)}$ um dinamarquês, ${ }^{(21)}$ um canadense, ${ }^{(22)}$ um de nacionalidade árabe, ${ }^{(23)}$ um espanhol, ${ }^{(24)}$ e um proveniente de Singapura ${ }^{(25)}$.
Dessa amostra, três deles foram selecionados do banco de dados BVS ${ }^{(15,16,26)}$ (sendo dois em base Scielo ${ }^{(15,26)}$ e outro em LILACS ${ }^{(16)}$ um em base de dados CINAHL, ${ }^{(19)}$ dois em Scopus, ${ }^{(22,23)}$ quatro em PsycINFO,(17,18,20,27) um no portal Pubmed, ${ }^{(24)}$ e dois em base Embase. ${ }^{(21,25)}$ Em relação ao ano de publicação online, houve um de 1984, ${ }^{(22)}$ um de 1992,(18) um de 1994, ${ }^{(19)}$ um de 2005, ${ }^{(17)}$ dois de 2008, ${ }^{(16,26)}$ um de $2011,{ }^{(15)}$ dois de $2012{ }^{(20,27)}$ um de $2013,^{(24)}$ um de $2015^{(21)}$ e dois de 2016. ${ }^{(23,25)}$

Quanto ao tipo de estudo, um é quantitativo, ${ }^{(17)}$ quatro são quanti-qualitativos, ${ }^{(15,19,24,27)}$ e o restante qualitativos, ${ }^{(16,18,20-26)}$ sendo realizados com abordagens variadas por meio de observação, estudo de caso, questionários, entrevistas ou grupos focais.

De forma geral, as pesquisas objetivaram conhecer as percepções maternas sobre o desenvolvimento infantil, com foco no desenvolvimento emocional, e identificar se as orientações recebidas também se focaram nessa área. Os estudos envolveram percepções de mulheres que já haviam sido mães e amamentaram, e mães que estavam amamentando no momento da pesquisa. Não houve delimitação de idade para as crianças amamentadas.

Quanto à análise de conteúdo dos estudos, emergiram as seguintes categorias: percepções e conhecimentos sobre amamentação, percepções sobre fatores que podem influenciar o desmame, percepções sobre fatores que podem influenciar na manutenção da amamentação, percepções e sentimentos resultantes da amamentação, percepções sobre influência da amamentação no crescimento e desenvolvimento infantil e percepções sobre as orientações recebidas pelos profissionais.

Percepções e conhecimentos sobre amamentação

Os estudos mostram uma variação no conhecimento sobre a amamentação: algumas mães desconheciam o conceito de "amamentação exclusiva"(23) ou não sabiam a real diferença na escolha entre a opção pela fórmula infantil ou leite materno. ${ }^{(16)}$ Outras mostraram saber as propriedades do leite materno, bem como descrever as técnicas adequadas de amamentarem os seus bebês. ${ }^{(24)}$

O conhecimento prévio sobre a amamentação pode ter grande influência sobre a prática, e mães com maior conhecimento, maior escolaridade ou maior renda tendem a ter mais intenção de amamentar por período maior. ${ }^{(17,21,27)}$ 


\section{Percepções sobre fatores que podem influenciar o desmame}

A maioria dos estudos mostrou nas falas das mães grande quantidade de fatores que influenciam na amamentação. Destaca-se nos estudos que as mães frequentemente percebem que o seu próprio leite é "fraco" ou "não sustenta". ${ }^{(16,20,21,23,25,26)}$ Essa percepção ocorre quando as mães ouvem os choros dos seus filhos, trazendo-lhes a sensação de que eles não estão recebendo o aporte necessário de calorias, pois o tempo de digestão é severamente mais rápido em comparação com as crianças que se alimentam por leite $\operatorname{artificial.~}^{(23)}$

Para algumas mães, estabelece-se um círculo vicioso, na medida em que quanto mais veem seus filhos chorando por uma aparente fome ou falta de saciedade mais ficam preocupadas e insatisfeitas e a produção do leite pode realmente estar diminuindo. (21) Frente a esse auto conceito, muitas vezes desistem da amamentação e oferecem leite artificial, ${ }^{(21,25)}$ muitas vezes com adição de alimentos ricos em carboidratos como cereais ou "engrossantes" que promovem rápida saciedade. ${ }^{(23)}$

Destaca-se também a influência das avós, tanto paternas quanto maternas que, ao verem seus netos chorando, muitas vezes julgam o cuidado das mães como ineficiente e acabam por oferecer leite artificial. ${ }^{(23,26)}$ Além disso, destacam-se: preocupação com a aparência das mamas, ${ }^{(17)}$ dor e fissuras nos mamilos, ${ }^{(21,25,27)}$ vergonha de amamentar em público ${ }^{(24)}$ sobretudo quando o filho já é maior de um ano, tem maior estatura e consegue andar. ${ }^{(19,25)}$

As condições trabalhistas também são apontadas como fatores que influenciam na amamentação. Há falta de apoio por parte das chefias durante o dia de trabalho para que a mãe se ausente por um breve período para amamentar e inexistem salas dentro do ambiente de trabalho para realizar a ordenha para manutenção da lactação. ${ }^{(25,26)}$

As mães apontam ainda que se sentem inseguras e ressentidas, podendo diminuir o tempo de amamentação, por não conseguirem realizar na prática a imagem que lhes é imposta por propagandas na mídia e há uma diferença entre "amamentação ideal" e "amamentação real". ${ }^{(21,22,26)}$

\section{Percepções sobre fatores que podem influenciar na manutenção da amamentação}

Mesmo diante de tantas dificuldades, verificou-se que quanto maiores as atitudes positivas maternas em relação à amamentação já durante a gestação, suas intenções de amamentar e o incentivo de pessoas próximas, maiores também as chances de manterem a amamentação por mais tempo. ${ }^{(17)}$

O companheirismo, o apoio e o incentivo do pai podem influenciar diretamente na manutenção da amamentação. ${ }^{(22,25,26)}$ Além disso, mulheres que amamentaram o primeiro filho, tendem a amamentar por mais tempo o segundo. ${ }^{(18)}$

\section{Percepções e sentimentos resultantes da amamentação}

Quando analisadas as falas das mães percebe-se que há um contraste entre sentimentos positivos e negativos em relação à prática de amamentar. Algumas mães sentem-se completas e satisfeitas ao amamentar, e outras podem sentir-se culpadas por terem desistido ou não terem conseguido manter a amamentação.

As mães que conseguiram manter a prática ou ao menos amamentar por algum tempo referem ser uma experiência positiva por passarem carinho, segurança, proteção e amor. . $^{(15,16,19,22,24-27)}$ Dizem se sentir orgulhosas de si próprias e satisfeitas, ${ }^{(22,24)}$ mais íntimas de seus filhos, ${ }^{(15,26)}$ além de considerarem um momento intenso, diferenciado e especial para a criação de vínculo entre mãe e filho ${ }^{(15,16,19,27)}$ e reconhecem ser um possível agente capaz de atenuar outras situações estressantes. ${ }^{(19)}$

Já quando uma mãe se vê impossibilitada de amamentar, seja por um motivo de saúde ou pela sua própria decisão, ela se sente culpada, relatando um "vazio" aparente ${ }^{(15)}$ e referindo ser mal interpretada e julgada pela sociedade como incapaz de ser mãe por não ter tido a persistência que outras tiveram..$^{(22,25)}$

\section{Percepções sobre influência da amamentação no crescimento e desenvolvimento infantil}

Entre os estudos que verificaram a percepção das mães sobre a influência da amamentação no desen- 
volvimento infantil, destacam-se os relatos de que as motivações para as mães amamentarem são por saberem que é mais saudável e natural para seus filhos, favorece o crescimento mais adequado da criança e desenvolvimento do seu sistema imunológico, do sistema ósseo e do cérebro, previne agravos à saúde, além da proporção de nutrientes mais equilibrada do leite materno quando comparado ao leite artificial. Elas reconhecem que o leite materno atua como um facilitador do funcionamento do sistema gastrointestinal, diminuindo as cólicas e alergias, e favorecendo a digestão. ${ }^{(15,17,19,23,26,27)}$ Essas publicações evidenciam que as mães conhecem sobre os benefícios da amamentação para o desenvolvimento físico das crianças.

Com relação ao desenvolvimento emocional relatam que um dos motivos para manter a amamentação é para auxiliar a criança a ser mais segura, contente e madura. ${ }^{(19)}$

\section{Percepções sobre as orientações recebidas pelos profissionais}

Ao se tratar da prática de profissionais da área materno-infantil, algumas mães se sentem felizes ao perceberem que suas incapacidades eram menores do que esperavam, como quando a mãe quer desistir de amamentar por estar com fissura no mamilo, porém ela é auxiliada com técnicas capazes de curar sua dor. ${ }^{(25)}$ Entretanto, há muito mais percepções maternas de caráter negativo a respeito das orientações recebidas pelos profissionais.

A maioria das orientações recebidas pelos profissionais retratadas na literatura foram confusas, de modo que cada profissional demonstrava uma maneira diferente de auxiliar as mães, muitas vezes, tornando o processo de amamentação pouco individualizado, atuando de forma mecânica em demasia, ${ }^{(21,22,26,27)} \mathrm{e}$ incentivando até mesmo o uso da mamadeira de forma abusiva e muitas vezes desnecessária. ${ }^{(20)}$

Não se encontrou relatos sobre orientações de profissionais sobre o impacto da amamentação no desenvolvimento emocional das crianças.

Partindo do pressuposto de que o conhecimento em saúde é primordial para melhorias na manutenção da amamentação de forma global, o presente estudo observou a escassez de publicações nessa esfera específica, ainda que ela seja de extrema relevância. Junta- mente a isso, têm-se profissionais que em sua maior parte são despreparados não apenas por não atribuírem à criança um cuidado integral individualizado, mas por muitas vezes não terem conhecimento científico necessário do impacto da amamentação no cuidado à saúde, e por consequência, não conseguirem difundi-lo às mães.

\section{Conclusão}

Quanto à análise de conteúdo dos estudos, emergiram as seguintes categorias: percepções e conhecimentos sobre amamentação, percepções sobre fatores que podem influenciar o desmame, percepções sobre fatores que podem influenciar na manutenção da amamentação, percepções e sentimentos resultantes da amamentação, percepções sobre influência da amamentação no crescimento e desenvolvimento infantil e percepções sobre as orientações recebidas pelos profissionais. Esse estudo apresentou como as mães percebem a amamentação, seus conhecimentos e suas dificuldades e potencialidades que estimulam o desmame ou a manutenção desta prática. Também demonstrou os sentimentos, sejam eles de alegria e satisfação em conseguir amamentar ou culpa por não terem iniciado ou mantido a amamentação. As percepções sobre influência da amamentação no crescimento e desenvolvimento infantil e percepções sobre as orientações recebidas pelos profissionais também foram relatadas. É notável o impacto das orientações profissionais nas percepções maternas sobre a amamentação. Encontrou-se um único estudo sobre as percepções maternas sobre a influência da amamentação no desenvolvimento emocional infantil. Assim, a influência da amamentação no desenvolvimento emocional das crianças pode ser uma vantagem a mais para compor as orientações de incentivo à amamentação, pois o maior vínculo afetivo entre mãe e criança, promovido pela amamentação, pode favorecer o desenvolvimento emocional infantil, com impacto inclusive a longo prazo. As orientações devem enfocar os benefícios do aleitamento materno e considerar possíveis dificuldades inerentes a esta prática. Para futuras pesquisas, sugere-se a realização de estudos que apresentem de forma focada as percepções maternas sobre a influência da amamentação no desenvolvimento emocional de seus filhos. Por meio 
desses, espera-se que os pesquisadores e profissionais de saúde se sensibilizem para melhorar as orientações sobre a amamentação e seu impacto na promoção do desenvolvimento integral infantil.

\section{Referências}

1. Lamberti LM, Zakarija-Grkovi I, Walker CL, Theodoratou E, Nair H, Campbell H, et al. Breastfeeding for reducing the risk of pneumonia morbidity and mortality in children under two: a systematic literature review and meta-analysis. BMC Public Health. 2013;13(Suppl3):S18.

2. Victora CG, Bahl R, Barros AJ, França GV, Horton S, Krasevec J, et al. Breastfeeding in the 21st century: epidemiology, mechanisms, and lifelong effect. Lancet. 2016;(10017)387:475-90.

3. Lamberti LM, Walker CL, Noiman A, Victora CG, Black RE. Breastfeeding and the risk for diarrhea morbidity and mortality. BMC Public Health. 2011;13(Suppl3):S15.

4. Rollins NC, Bhandari N, Hajeebhoy N, Horton S, Lutter CK, Martines JC, et al. Why invest, and what it will take to improve breastfeeding practices? Lancet. 2016;(10017)387:491-504.

5. Dias IS, Correia S, Marcelino P. Desenvolvimento na primeira infância: características valorizadas pelos futuros educadores de infância. Rev Eletrônica Educ. 2013;7(3):9-24.

6. Girard LC, Doyle 0, Tremblay RE. Breastfeeding, cognitive and noncognitive development in early childhood: a population study. Pediatrics. 2017;139(4):e20161848.

7. Greenspan S. Clinical assessment of emotional milestones in infancy and early childhood. Pediatr Clin North Am. 1991;38(6):1371-85.

8. Freud S. Instintos e suas vicissitudes. $14^{\mathrm{a}}$ ed. In: Edição Standard brasileira das obras completas de Sigmund Freud. Rio de Janeiro: Imago Editora; 1972. p. 117-44.

9. Brazelton TB, Greenspan S. As necessidades essenciais das crianças - 0 que toda criança precisa para crescer, aprender e se desenvolver. Trad. Porto Alegre: Artmed; 2002.

10. Britton JR, Britton HL, Gronwaldt V. Breastfeeding, sensitivity, and attachment. Pediatrics. 2006;118 (5):e1436- 43.

11. Kim P, Feldman R, Mayes LC, Eicher V, Thompson N, Leckman JF, et al. Breastfeeding, brain activation to own infant cry, and maternal sensitivity. J Child Psychol Psychiatry. 2011;52(8):907-15
12. Lind JN, Li R, Perrine CG, Schieve LA. Breastfeeding and later psychosocial development of children at 6 years of age. Pediatrics. 2014;134(Suppl1):S36-S41.

13. Venancio SI, Escuder MM, Saldiva SR, Giugliani ER. A prática do aleitamento materno nas capitais brasileiras e Distrito Federal: situação atual e avanços. J Pediatr. 2010;86(4):31724.

14. Jager E, Skouteris H, Broadbent J, Amir L, Mellor K. Psychosocial correlates of exclusive breastfeeding: a systematic review. Midwifery. 2013;29(5):506-18.

15. Carrascoza KC, Possobon RF, Costa-Júnior AL, Moraes AB. Aleitamento materno em crianças até os seis meses de vida: percepção das mães. Physis. 2011;21(13):1045-59.

16. Faustino-Silva DD, Lima DL, Rosito DB, Ribeiro SM, Figueiredo MC. Percepções e saberes de um grupo de gestantes sobre aleitamento materno-um estudo qualitativo. RF0. 2008;2(13):7-11.

17. Coutinho J, Leal IP. Atitudes de mulheres em relação à amamentação-estudo exploratório. Aná Psicológica. 2005;3(XXIII):277-82.

18. Epstein K. The interactions between breastfeeding mothers and their babies during the breastfeeding session. Early Child Dev Care. 1993;87(1):93-104.

19. Hills-Bonczyk SG, Tromiczak KR,Avery MD, Potter S, Savik K, Duckett ப. Women's experiences with breastfeeding longer than 12 months. Birth. 1994;21(4):206-12.

20. Redshaw M, Henderson J. Learning the hard way: expectations and experiences of infant feeding support. Birth. 2012;39(1):21-9.

21. Kronborg $\mathrm{H}$, Harder I, Hall E. First time mothers' experiences of breastfeeding their newborn. Sex Reprod Healthc. 2015;6(2):82-7.

22. Hewat RJ, Ellis DJ. Breastfeeding as a maternal child team effort: Women's perceptions. Health Care Women Int. 1984;5(5-6):437-52.

23. Radwan H, Sapsford R. Maternal perceptions and views about breastfeeding practices among emirati mothers. Food Nutr Bull. 2016;37(1):73-84.

24. Díaz-Meneses G. Breastfeeding: an emotional instinct. Breastfeed Med. 2013;8(2):191-7.

25. Choo PJ, Ryan K. A qualitative study exploring first time mothers' experiences of breastfeeding in Singapore. PoSH. 2016;25(1):5-12.

26. Machado MM, Bosi ML. Compreendendo a prática do aleitamento exclusivo: um estudo junto a lactantes usuárias da rede de serviços em Fortaleza, Ceará, Brasil. Rev. Bras. Saúde Mater. Infant. 2008; 8(2):187-96.

27. Cunha AC, Santos C, Gonçalves RM. Concepções sobre maternidade, parto e amamentação em grupo de gestantes. Arq Bras Psicol. 2012;64(1):139-55. 